\title{
DESPLIEGUE AUTOMÁTICO DE LABORATORIOS REMOTOS EXTENDIENDO LAS CAPACIDADES DE EJSS
}

\author{
Iñigo Aizpuru Rueda \\ Airbus Defence \& Space, Getafe, Madrid, \\ inigo.aizpuru@airbus.com
}

Eva Besada Portas, Jesús Chacón, José Antonio López-Orozco

Departamento de Arquitectura de Computadores y Automática. Universidad Complutense de Madrid (UCM), \{ebesada, jeschaco, jalopez\}@ucm.es

\section{Resumen}

Este artículo presenta una nueva metodología para automatizar el proceso de puesta a punto y despliegue de los laboratorios remotos que involucran en su diseño a EJsS. La propuesta consiste en extender la funcionalidad y adaptar la interfaz de EJsS mediante una infraestructura autocontenida y distribuible de Plugins que pueden ser fácilmente activados y desactivados, según las necesidades del usuario. Además, muestra como los Plugins pueden ser utilizados para facilitar la creación de la interfaz gráfica de un laboratorio particular, gestionar su conexión con los laboratorios remotos disponibles, automatizar el despliegue del laboratorio, y centralizar la codificación y compilación de la aplicación que cierra el lazo de control sobre la planta. La herramienta disponible al final de este proceso es una solución centralizada, compacta, de bajo coste y código abierto para que los docentes desarrollen y desplieguen fácilmente sus laboratorios remotos.

Palabras clave: Laboratorios Remotos, EjsS, Node.js, Raspberry PI, Despliegue Automático

\section{INTRODUCCIÓN}

La experiencia en el laboratorio es fundamental en la enseñanza de las ingenierías y ciencias empíricas, ya que aporta al estudiante un nexo de unión entre los conceptos teóricos que se abordan en el aula, y la manifestación de éstos en el mundo real $[11,21]$. Sin embargo, los costes de creación de los laboratorios, y las limitaciones de espacio y personal técnico especializado para mantenerlos, impiden que, en muchas ocasiones, las instituciones educativas dispongan de la infraestructura necesaria para proporcionar a los estudiantes un entorno de experimentación adecuado [16].

Por este motivo, muchas de estas instituciones dedican sus esfuerzos al desarrollo de laboratorios online, una solución alternativa que incluye a los laboratorios virtuales (que dan acceso a

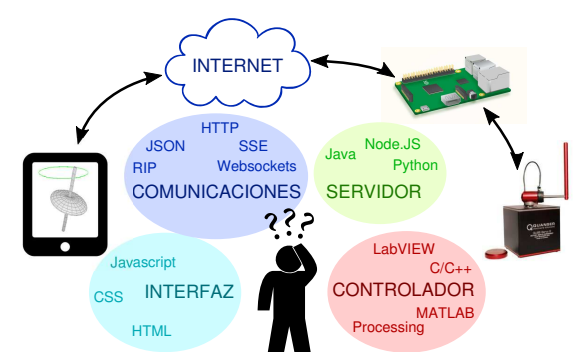

Figura 1: Dificultad del proceso de puesta en marcha de un laboratorio online.

través de Internet a herramientas interactivas de simulacion), remotos (a sistemas reales) y mixtos (que explotan las ventajas de ambos enfoques) $[4,14,19,25]$. Además, es importante destacar que estudios recientes avalan, desde un punto de vista educativo, el uso de este tipo de laboratorios, ya que aportan los mismos beneficios que los laboratorios in-situ, al tiempo que proporcionan ventajas adicionales como la reducción de costes, el aumento de las horas de disponibilidad del material del laboratorio y la seguridad en su uso $[13,15,17,20,22,23]$.

Sin embargo, el desarrollo de estas herramientas no es trivial, ya que involucran muchos aspectos de diseño y problemática tecnológica propia (como el acceso al hardware, la gestión de las comunicaciones, etc) del área de las Tecnologías de la Información y de la Comunicación (TIC). Afortunadamente, estas necesidades suelen ser similares entre diferentes laboratorios online, a pesar de que la naturaleza del experimento pueda variar enormemente. Por ello, resulta conveniente abordar el diseño desde un punto de vista sistemático, que proporcione al educador herramientas de puesta en marcha y gestión de los laboratorios automáticas, para que pueda centrar sus esfuerzos en el diseño de la actividad experimental de su materia, y se olvide, en la medida de lo posible, de los problemas tecnológicos asociados.

En linea con lo anterior, es posible encontrar trabajos que proponen soluciones arquitecturales 
parar simplificar y reutilizar los desarrollos. Por ejemplo, [12] propone un protocolo de interoperabilidad con sistemas remotos (RIP, Remote Interoperability Protocol), que estandarizara la comunicación entre las aplicaciones software del laboratorio remoto, para racionalizar su desarrollo. Por otra parte, también se han realizado esfuerzos para lograr la integración robusta de los laboratorios online con plataformas de gestión del aprendizaje (LMS, Learning Management Systems) habituales (p.e. Moodle). Un ejemplo es la red de laboratorios remotos UNILabs [3], promovida desde la Universidad Nacional de Educación a Distancia (UNED) y en la que participan otras universidades nacionales e internacionales. Alternativamente, también han proliferado algunas metodologías más sencillas, de bajo coste y código abierto $[6,8,18]$, que facilitan la puesta en marcha de laboratorios remotos con una baja inversión económica y un alto grado de flexibilidad [7, 10]. Finalmente, es importante destacar la existencia de Easy Javascript Simulations (EJsS, [1]), una herramienta versátil para la creación de laboratorios virtuales, que es extensamente utilizada por la comunidad educativa de ciencias (tal y como se puede observar en el amplio catálogo de ejemplos disponibles en [1]).

Aunque las soluciones más complejas y robustas son un pilar fundamental de las instituciones de educación a distancia (como la UNED), en las universidades presenciales (como la de los autores, la Universidad Complutense de Madrid UCM), las necesidades educativas son diferentes. En primer lugar, las prácticas presenciales de laboratorio siguen ocupando un lugar importante, por lo que los laboratorios online son, más que una solución alternativa, un complemento a los recursos existentes. Además, las soluciones de bajo coste mencionadas pueden ser usadas para realizar demostraciones en vivo (o incluso experimentos colaborativos) en el aula, como complemento a las actividades tradicionales, para motivar al alumno y fomentar su interés por la asignatura.

A pesar de todos los avances realizados, las metodologías de bajo coste mencionadas adolecen de una automatización parcial, que hace que el docente deba realizar manualmente una parte del proceso general de despliegue y mantenimiento de sus laboratorios online. Para reducir el esfuerzo del docente en este ámbito, en este trabajo se presenta una nueva solución autónoma y autosuficiente (i.e. libre de infraestructuras adicionales complejas), completa y automatizada para el desarrollo y despliegue de laboratorios remotos.

Las primeras características son consecuencia del uso de Node.js (un entorno de desarrollo y eje- cución de aplicaciones distribuidas en tiempo real) para la creación del servidor del laboratorio y la Raspberry PI (un pequeño computador de bajo coste) para el despliegue del software del laboratorio remoto $[6,8]$. Las segundas se logran mediante la extensión de la funcionalidad de EJsS, de forma que éste se convierta en la herramienta desde la que el docente lleve a cabo la mayor parte de las actividades de puesta en marcha y gestión de sus laboratorios remotos.

Finalmente, y no por ello menos importante, es importante destacar que la metodología presentada en este trabajo para extender la funcionalidad de EJsS puede ser utilizada para personalizar el funcionamiento de EJSS para otros tipos de laboratorios u otras labores diferentes.

La estructura del resto del documento es la siguiente. La sección 2 presenta las características más relevantes de EjsS, la herramienta elegida para centralizar el desarrollo y despliegue de los laboratorios virtuales y remotos, y la metodología propuesta para extender sus capacidades. La sección 3 muestra la nueva herramienta desarrollada en este trabajo, destacando los beneficios obtenidos por la extensión de EJsS, y por el uso de Node.js y la Raspberry PI para soportar las funcionalidades genéricas más relevantes del laboratorio remoto. Finalmente, la sección 4 expone las conclusiones del trabajo realizado.

\section{EXTENDIENDO EJsS MEDIANTE PLUGINS}

\subsection{LA HERRAMIENTA EJsS}

Easy Javascripts Simulations (EJsS, [1]) es una herramienta gratuita y de código abierto que facilita la creación de simulaciones físicas interactivas a usuarios (p.e. docentes, alumnos) con conocimientos limitados de programación. En esencia, se trata de una herramienta gráfica que permite al usuario definir, de forma desacoplada, el Modelo de la simulación mediante el uso de Ecuaciones Diferenciales Ordinarias (ODEs) y la Vista que conforma su representación gráfica e interactiva. Además, permite al usuario generar el código (inicialmente Java, actualmente HTML + Javascript) y la aplicación (applet o página web) correspondiente. De serie, EJsS incorpora diversos métodos numéricos de integración, la capacidad de gestión de eventos y otra serie de mecanismos sofisticados para la creación de simulaciones complejas. También dispone de una amplia paleta de componentes visuales y elementos interactivos que ayudan a sus usuarios a diseñar entornos gráficos ilustrativos y atractivos de sus simulaciones. Por to- 


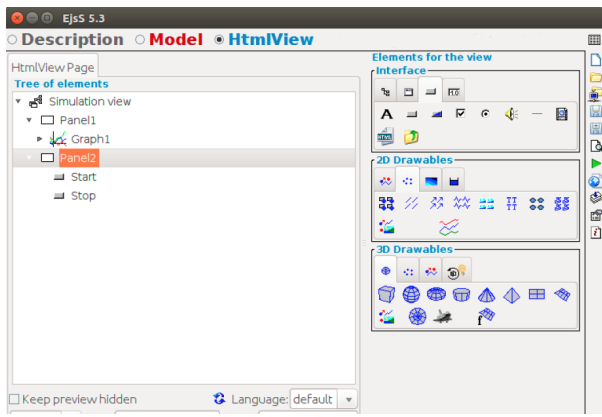

(a) Panel de la vista

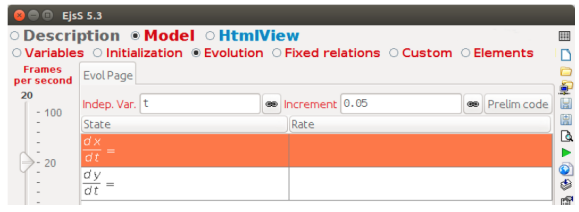

(b) Panel del modelo

Figura 2: Interfaz gráfica habitual de EJsS

dos estos motivos resulta una herramienta ideal para la creación de laboratorios virtuales.

Gracias a su clara separación entre el Modelo y la Vista, a su facilidad de manejo y a sus capacidades gráficas, el uso de EJsS se ha extendido a áreas para las que no fue inicialmente concebido. En particular, dentro del ámbito de los laboratorios online, es importante destacar su uso como herramienta de definición de la Vista con la que los alumnos interactúan y observan el comportamiento de numerosos laboratorios remotos $[6,7,8,9,24]$. Otro factor decisivo, favorecedor de esta expansión, es la posibilidad que un usuario tiene de extender (en parte, y tal y como se describe a continuación) la funcionalidad de EJsS.

\subsection{EXTENSIONES ESTÁNDAR}

Actualmente existen tres formas diferentes de añadir o modificar la funcionalidad de EJsS:

- Mediante la incorporación de Elementos a las paletas de diseño de la Vista (representadas a la derecha de la Figura 2a). Estos nuevos Elementos gráficos pueden usarse en el entorno gráfico de la aplicación del usuario de forma análoga a la que se incorporan los elementos disponibles por defecto en EJsS. Para crearlos, el usuario debe: copiar el nodo del árbol de elementos visuales que contiene la estructura que desea guardar, pegarlo dentro de la pestaña de elementos compuestos del grupo Interfaz, y darle un nombre e icono representativo.

- Mediante la incorporación de componentes en la pestaña de Elementos del Modelo (en la parte

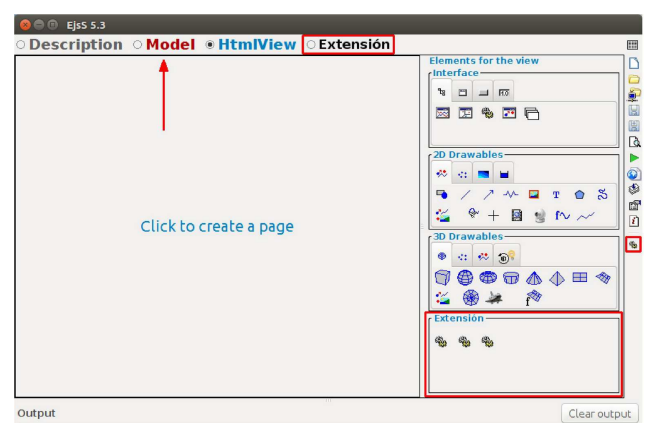

(a) Extensiones de botones, menú principal y Vista

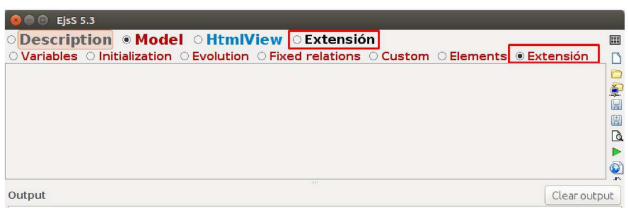

(b) Extensión de las pestañas del Modelo

Figura 3: Extensiones de EJsS mediante Plugins

superior-derecha de la Figura 2b). Estos Elementos generalmente encapsulan librerías externas o añaden pequeñas funcionalidades. Para añadirlos, sólo hay que almacenar la librería correspondiente y unos ficheros auxiliares (iconos, ayuda) en un directorio concreto de EJsS.

- Modificando directamente el código de EJsS, ya que es una herramienta de código abierto y se encuentra disponible en un repositorio público.

Las dos primeras opciones son adecuadas cuando los Elementos de la Vista o del Modelo se encuentran desacoplados de la herramienta EJsS y no requieren mucha integración con ella. Por ejemplo, para reutilizar la representación gráfica de un dispositivo (un tanque de agua, un sensor de nivel, etc) resulta conveniente crear un nuevo Elemento de la Vista para dicho objeto. O para facilitar la comunicación entre la aplicación desarrollada con EJsS y una aplicación externa (p.e. el servidor de un laboratorio remoto) es útil crear una librería y encapsularla en un Elemento del Modelo. Sin embargo, estos mecanismos no permiten personalizar ni ampliar la propia interfaz de EJsS.

En cuanto a la tercera aproximación, aunque evidentemente ofrece una flexibilidad y control absoluto, tampoco es adecuada para todos los escenarios. Por una parte, introducir cambios en el código que da soporte a EJsS requiere un conocimiento profundo del mismo debido a su complejidad y tamaño. Por otra, no es conveniente tener que modificar el código de la herramienta cada vez que existe una nueva versión de la misma o se desea añadirle nuevas características. 
Los motivos mencionados sugieren la necesidad de un nuevo mecanismo que permita adaptar fácilmente la interfaz y la funcionalidad de EJsS a las necesidades cambiantes de sus posibles usuarios. A continuación se presenta una estrategia elegante a esta cuestión.

\section{$2.3 \quad$ NUEVAS EXTENSIONES MEDIANTE PLUGINS}

El mecanismo de extensión propuesto en esta trabajo trata de satisfacer los siguientes objetivos:

- Distribución sencilla, de forma que se facilite la obtención e instalación de una determinada extensión de EJsS.

- Autocontenido, de forma que no se requieran instalaciones o acciones adicionales por parte del usuario.

- No intrusivo, para que pueda ser eliminado por completo, sin dejar trazas, cuando sea necesario.

Para alcanzarlos, se propone un mecanismo que agrupa un conjunto de modificaciones en una Infraestructura de Extensiones (Plugins). Un Plugin puede verse como una generalización de un Elemento del Modelo o de la Vista, que permite: 1) agrupar y cohesionar la adición de diferentes componentes (Elementos del Modelo, Elementos de la Vista y otras funcionalidades que veremos a continuación) y 2) definir, como principal novedad, una interfaz gráfica personalizada que adapte EJsS a las necesidades de sus usuarios.

Para poner a prueba las ventajas de esta nueva estrategia, se ha modificado el código de EJsS (de la forma menos intrusiva posible) para que se pueda realizar la extensión automática mediante Plugins en los puntos de interés que a continuación se explican. Además, los cambios que cada uno de los tipos de extensiones permitidas producen sobre el entorno gráfico de EJsS pueden observarse (enmarcados en rojo) en la Figura 3, yuxtapuesta y situada a la derecha de la Figura 2 que muestra la interfaz gráfica habitual de EJsS. Los tipos de extensión implementados actualmente se dan en:

- La Barra de Botones de EJsS, situada en el lateral derecho de EJsS y cuyos elementos por defecto sirven para realizar (al ser pulsados) una acción programada (como crear la aplicación de la simulación o salvar su contenido) o desplegar menús contextuales que agrupan un número de acciones que el usuario debe seleccionar. De esta forma, los Plugins añaden nuevos botones de ambos tipos, de forma que el usuario pueda ejecutar fácilmente acciones alternativas, necesarias para la extensión que tiene instalada.
- La Barra de Opciones Principales de EJsS, situada en la parte superior de EJsS y donde por defecto se desacopla la definición del Modelo y de la Vista. Así, los Plugins resaltan (en el nivel superior de definición de EJsS) la funcionalidad más relevante de una extensión.

- Los Grupo de Controles de la Vista, situados dentro del Panel de la Vista y donde por defecto se muestran los elementos gráficos disponibles. En este caso, los Plugins reunen, en un grupo propio de la extensión, elementos visuales habituales y su funcionalidad correspondiente.

- Las Pestañas del Modelo situadas dentro del Panel del Modelo y donde por defecto se muestran las pestañas que EJsS utiliza habitualmente para modelar un sistema. En este caso, los Plugins sitúan, fuera de la pestaña de Elementos del Modelo estándar de EJsS, funcionalidad relevante (a un nivel intermedio) para el funcionamiento de la extensión.

Además, para facilitar la distribución, instalación y desistalación de la funcionalidad de un Plugin se sigue una estrategia similar a la de los Elementos del Modelo. Es decir, cada Plugin es una librería de java que incorpora los métodos y funcionalidad deseada para una determinada extensión, y que el usuario debe situar en un directorio determinado de la herramienta EJsS, para que pueda ser detectado y cargado durante el arranque de la misma.

Finalmente, conviene resaltar de nuevo la diferencia metodológica que existe entre la nueva propuesta de extensión y la estándar: mientras que los Elementos habituales tienen un ámbito concreto y limitado de extensión, los Plugins permiten adaptar la interfaz gráfica de EJsS para una aplicación determinada y añadir nuevas funcionalidades en los puntos de interés adecuados de EJsS.

\section{DESPLIEGUE AUTOMÁTICO DE UN LABORATORIO REMOTO DE LA UCM}

En esta sección se describe el laboratorio elegido y las automatizaciones alcanzadas al aplicar la nueva metodología de Plugins de EJsS.

\subsection{EL LABORATORIO ELEGIDO}

El laboratorio remoto elegido es una solución compacta, de bajo coste y de código abierto, esquematizada en la Figura 4 y detallada en [6, 8]. Utiliza Node.js para programar y ejecutar el servidor de páginas web (de acceso, menú, ayuda, datos e interfaz gráfica de la experiencia) del laboratorio y 


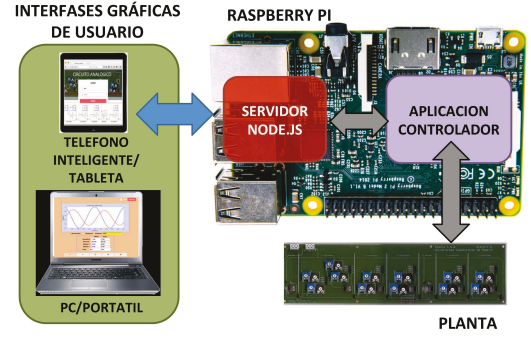

(a) Hardware del laboratorio y distribución de las aplicaciones

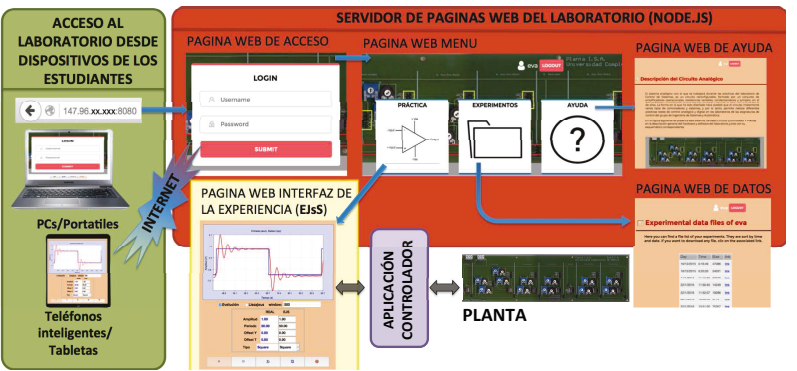

(b) Software y páginas web del laboratorio

Figura 4: El laboratorio remoto de bajo coste y código abierto de la UCM

una Raspberry PI para alojar al servidor y al controlador (programado en $\mathrm{C}$ y responsable de cerrar el lazo de control sobre la planta). Además, la pagina web de la interfaz de la experiencia (enmarcada en amarillo en la Figura 4b, y usada por los alumnos para visualizar la evolución de las variables de la planta y modificar los parámetros del controlador) es generada desde EJsS.

Aunque las tecnologías utilizadas y la metodología seguida son usadas con éxito, desde 2016, en varios laboratorios remotos de la UCM, la creación de nuevas prácticas requiere que el docente:

- Adapte un proyecto EJsS básico a las características de cada práctica, genere la página web correspondiente al proyecto modificado, rectifique manualmente el código generado por la herramienta, y descargue el mismo sobre el directorio correspondiente de la Raspberry PI.

- Edite, modifique y compile, el código del controlador que cierra el lazo sobre la planta disponible en cada laboratorio.

- Edite y adapte el código del servidor de la página web del laboratorio a las variables que la pagina web generada con EJsS debe intercambiar con el código del controlador.

Es decir, el profesor debe utilizar diferentes herramientas (EJsS, editores, compiladores) en diferentes plataformas (su ordenador, la Raspberry PI) para realizar el despliegue de cada nueva experiencia, divergiendo durante este tedioso proceso de su objetivo primordial: poner a la disposición de los alumnos una nueva experiencia educativa. Tal y como veremos a continuación, centralizar y automatizar las labores previas en EJsS facilitará la labor y reducirá la carga de trabajo del docente.

\subsection{EL PLUGIN DESARROLLADO}

Los objetivos concretos del Plugin de EJsS desarrollado para el laboratorio seleccionado son:
- Facilitar la creación y el mantenimiento de los interfaces habituales del laboratorio.

- Automatizar completamente el despliegue de la interfaz gráfica del laboratorio.

- Automatizar y centralizar el proceso de edición y compilación del código fuente que cierra el lazo de control sobre la planta.

- Gestionar las conexiones entre la interfaz del laboratorio y los laboratorios remotos disponibles.

Para lograrlos se han creado, e incorporado en un único Plugin, las siguientes extensiones:

- Un nuevo Grupo de Controles de la Vista (visibles en la parte inferior derecha de la Figura 5a) que tienen como objeto facilitar la creación de los elementos gráficos habituales (representados en la Figura 5b) del laboratorio seleccionado. Más en concreto, los elementos gráficos de 호 permiten la autenticación de un usuario (con su usuario y contraseña) en un laboratorio remoto; los de 盟, configurables automáticamente a través de un mensaje JSON del servidor, permiten a la interfaz del laboratorio interactuar con las señales existentes en el laboratorio; y los

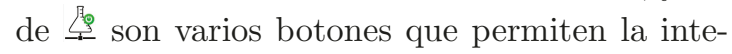
raccion con el laboratorio remoto. Por lo tanto, esta extensión agiliza la puesta en marcha de un nuevo laboratorio remoto, ya que evita que el docente tenga que añadir, configurar y programar la funcionalidad de cada uno de los elementos gráficos habituales por separado.

- Un nuevo botón (observable en la parte inferior de la barra de botones de la Figura 6a) que, desplegando el menú mostrado en la Figura $6 \mathrm{~b}$, permite a un usuario de EjsS descargar automáticamente en el servidor de la Raspberry PI la nueva interfaz de laboratorio. Además, para realizar la descarga, lleva a cabo las labores de manipulación del código de EJsS que anteriormente realizaba de forma manual el docente que 


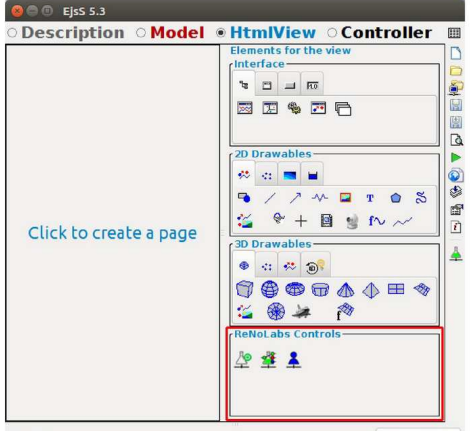

(a) Extensión de elementos gráficos

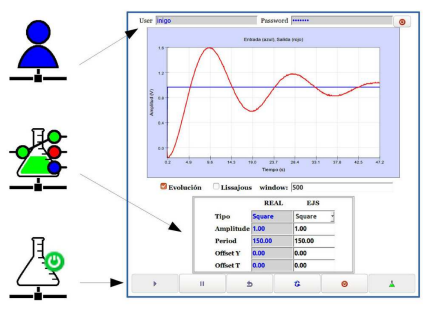

(b) Utilidad de los elementos gráficos

Figura 5: Nuevas herramientas gráficas

ponía en marcha el laboratorio. Es decir, esta extensión automatiza el proceso de despliegue del código de la página web de la interfaz gráfica del laboratorio (mostrada en la Figura 5b) en la Raspberry PI.

- La pestaña Controlador de la Barra de Opciones Principales de EJsS (cuyo contenido se observa en la Figura 7) y que sirve para descargar, en EJsS, el código fuente (en C) del controlador de la planta que se encuentra almacenado en la Raspberry PI, editarlo, enviarlo de nuevo a la Raspberry PI y compilarlo. Además, durante la descarga y el envío, despliega un menú en el que se requiere la identificación del usuario, para que la herramienta pueda ser utilizada por los alumnos para descargar y ejecutar sus propias versiones del código del controlador en la Raspberry PI. Por lo tanto, esta extensión centraliza en EJsS la gestión de la aplicación que cierra el lazo de control sobre la planta.

- La pestaña Laboratorio Remoto (visible en la Figura 8 dentro de las opciones del Modelo) que sirve para conectar la página web de la experiencia (generada con el botón de descarga de la extensión) con los laboratorios remotos disponibles en diferentes Raspberry PI. Más en concreto, las variables que definen cada conexión (dos en el ejemplo de la figura) dan acceso a métodos y atributos que permiten que la página web de la experiencia conozca el estado de los laboratorios remotos en cualquier instante. Además, es importante aclarar que

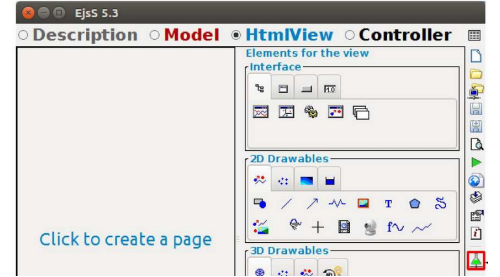

(a) Nuevo botón de descarga

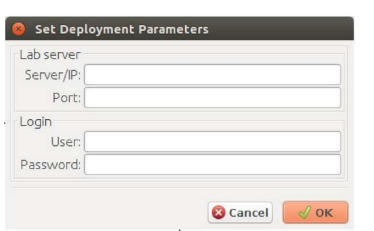

(b) Menú asociado al botón de descarga

Figura 6: Herramienta de descarga

para poder establecer la relación entre los con-

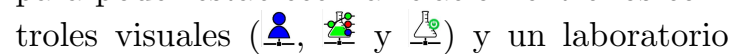
remoto concreto, la variable de dicho laboratorio debe ser suministrada como parámetro de configuración de dicho elemento gráfico. Por lo tanto, esta extensión permite gestionar la conexión entre la interfaz del laboratorio y los laboratorios remotos disponibles, y da soporte a la funcionalidad requerida por la extensión del grupo de controles de la Vista.

Conviene señalar que para dar soporte a las interacciones necesarias entre EJsS y el servidor Node.js del laboratorio remoto, éste último también ha tenido que ser modificado. Por lo tanto, su versión actual no solo da soporte a la página web del laboratorio, sino que también responde a las interacciones requeridas por el Plugin desarrollado para poner en marcha un nuevo laboratorio remoto.

Además, la interacción del alumno con el laboratorio es similar a la que se describe en [8], ya que el trabajo aquí presentado se ha centrado en simplificar las labores de los docentes, tanto durante la puesta en marcha del laboratorio elegido (por medio del desarrollo de un Plugin concreto) como durante la puesta en marcha de otros laboratorios (al haber dotado a EJsS con una nueva estructura de Extensiones aprovechable para adaptar dicha herramienta a diferentes necesidades).

Finalmente, pero no por ello menos importante, indicar que detalles adicionales sobre el mecanismo de Plugins presentado y sus posibilidades, y sobro otros ejemplos de uso pueden encontrarse en el Trabajo Fin de Master [5]. Además, el software desarrollado será puesto próximamente a disposición de la comunidad educativa en la sección de descargas de [2]. 


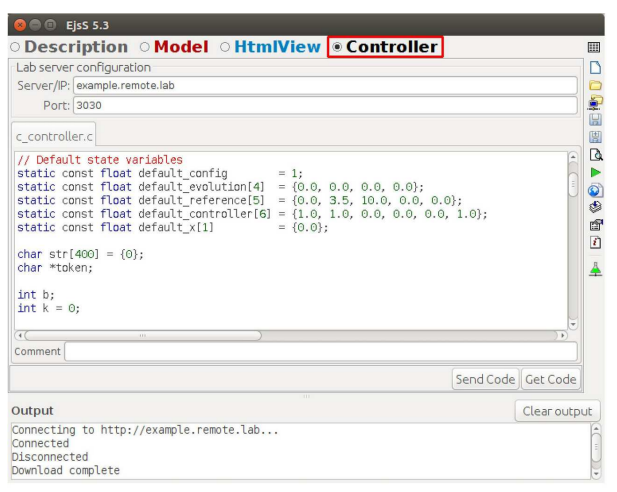

Figura 7: Herramienta para el Controlador en C

\section{CONCLUSIONES}

En este trabajo se presenta una nueva metodología para poder adaptar la interfaz gráfica de EjsS a las necesidades de una determinada aplicación e incorporar nuevas capacidades (elementos gráficos y funcionales, botones de acción y pestañas) en diferentes puntos de interés de dicha herramienta. El mecanismo desarrollado, una infraestructura de extensiones denominado Plugins, es no intrusivo, autocontenido y fácilmente distribuible.

Además, también se presentan las nuevas herramientas desarrolladas, sobre el nuevo mecanismo de Plugins de EJsS, para automatizar el proceso de puesta a punto de los laboratorios remotos de bajo coste y de código abierto de la UCM soportados por Node.js y la Raspberry PI. En concreto, las nuevas herramientas facilitan la creación de la interfaz gráfica del laboratorio y la gestión de su conexión con los laboratorios remotos disponibles, automatizan el despliegue del laboratorio, y centralizan la codificación y compilación del código del controlador.

\section{Agradecimientos}

Este trabajo ha sido parcialmente financiado por el Proyecto 139 de Innovación Educativa y Mejora de la Calidad Docente de la UCM. Los autores desean expresar su agradecimiento al Catedrático Francisco Esquembre, creador de la herramienta EJsS, por su ayuda y sugerencias durante el desarrollo del mismo.

\section{English summary}

\section{AUTOMATIC DEPLOYMENT OF REMOTE LABS BY EXTENDING EJsS CAPABILITIES}

\section{Abstract}

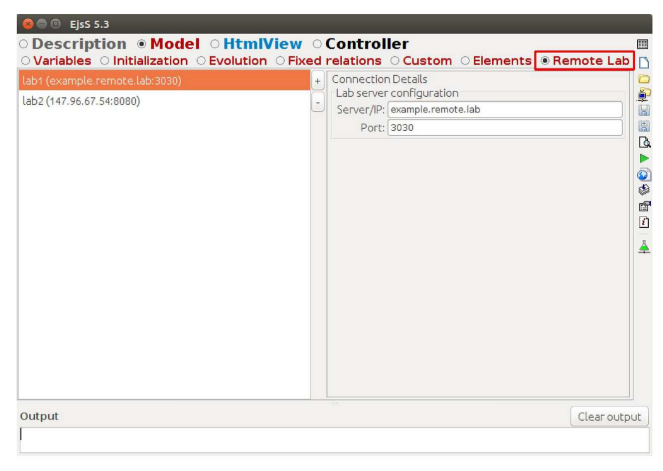

Figura 8: Pestaña del Laboratorio remoto

This paper presents a new methodology to automate the development and deployment process of remote laboratories that involve EJsS in their design. The proposal consists of extending the functionality and adapting the EJsS interface through a self-contained and distributable infrastructure of Plugins that can be easily activated and deactivated, according to the user needs. In addition, it shows how the Plugins can be used to accelerate the development of the graphical interface of a particular laboratory, manage its connection with the available remote laboratories, automate the deployment of the laboratory, and centralize the coding and compilation of the application which closes the control loop on the plant. The tool available at the end of this process is a centralized, compact, low cost and open source solution the helps teachers to easily develop and deploy their remote laboratories.

Keywords: Remote Laboratories, EJsS, Node.js, Raspberry PI, Automatic Deployment

\section{Referencias}

[1] EJsS Webpage. http://fem.um.es/Ejs.

[2] ISCARLab. www.dacya.ucm.es/isalab.

[3] UNILABs. https://unilabs.dia.uned.es.

[4] M. Abdulwahed and Z.K. Nagy. The TriLab, a novel ICT based triple access mode laboratory education model. Computers $\&$ Education., 56, 2011. 
[5] I. Aizpuru-Rueda. Generación y despliegue automático de laboratorios remotos con EjsS y Node.js. Master's thesis, Universidad Nacional de Educación a Distancia, 2018. cv4.ucm.es/moodle/course/view.php?id=4056.

[6] J. Bermudez-Ortega, E. Besada-Portas, J.A. Lopez-Orozco, J.A. Bonache-Seco, and J.M. de la Cruz. Remote web-based control laboratory for mobile devices based on EJsS, Raspberry $\mathrm{Pi}$ and Node.js. In IFAC Workshop on Internet Based Control Education, 2015.

[7] J. Bermúdez-Ortega, E. Besada-Portas, J. A. López-Orozco, and J. M. de la Cruz. A new open-source and smart-device accessible remote control laboratory. In Experiment@International Conference, 2017.

[8] E. Besada-Portas, J. Bermudez-Ortega, L. de la Torre, J.A. Lopez-Orozco, and J.M. de la Cruz. Lightweight Node.js EJsS-based web server for remote control laboratories. In 11th IFAC Symposium on Advances in Control Education, 2016.

[9] P. Casals-Torrens. Virtual laboratory for learning asynchronous motors in engineering degrees. IEEE Revista Iberoamericana de Tecnologias del Aprendizaje, 8(2), 2013.

[10] J. Chacon, J. Saenz, L. De La Torre, J.M. Diaz, and F. Esquembre. Design of a lowcost air levitation system for teaching control engineering. IEEE Access, 17(10), 2017.

[11] X. Chen, G. Song, and Y. Zhang. Virtual and remote laboratory development: A review. In 12th International Conference on Engineering, Science, Construction, and Operations in Challenging Environments, 2010.

[12] L. de la Torre, J. Chacon, and D. Chaos. Remote interoperability protocol specification, 2019. https://doi.org/10.5281/zenodo.2644242.

[13] L. de la Torre, J. P. Sanchez, and S. Dormido. What remote labs can do for you. Physics Today, 69:48-53, 2016.

[14] S. Dormido. Control learning: present and future. Annual Reviews in Control, 28:115$135,2004$.

[15] E.K. Faulconer and A.B. Gruss. A review to weigh the pros and cons of online, remote, and distance science laboratory experiences. The International Review of Research in Open and Distributed Learning, 19(2), 2018.
[16] L. Gomes. Current trends in remote laboratories. IEEE Transactions on Industrial Electronics, 56, 2009.

[17] C. Gravier, J. Fayolle, B. Bayard, M. Ates, and J. Lardon. State of the art about remote laboratories paradigms - foundations of ongoing mutations. International Journal of Online Engineering, 4(1), 2008.

[18] R. Heradio, J. Chacon, H. Vargas, D. Galan, J. Saenz, L. De La Torre, and S. Dormido. Open-source hardware in education: A systematic mapping study. IEEE Access, 6, 2018.

[19] R. Heradio, L. de la Torre, and S. Dormido. Virtual and remote labs in control education: A survey. Annual Reviews in Control, 42, 2016.

[20] N. Kostaras, M. Xenos, and A.N. Skodras. Evaluating usability in a distance digital systems laboratory class. IEEE Transactions on Education, 54(2), 2011.

[21] J. Ma and J.V. Nickerson. Hands-on, simulated, and remote laboratories: A comparative literature review. ACM Computing Surveys, 38(7), 2006.

[22] Z. Nedic, J. Machotka, and A. Nafalski. Remote laboratories versus virtual and real laboratories. In Frontiers in Education Conference, volume 1, 2003.

[23] J.V. Nickerson, J.E. Corter, Sven K. Esche, and C. Chassapis. A model for evaluating the effectiveness of remote engineering laboratories and simulations in education. Computers \& Education, 49(3), 2007.

[24] M. Reyes, S. Sanchez-Herrera, A. Mejias, M. Marquez, and J.M. Andujar. A fully integrated open solution for the remote operation of pilot plants. IEEE Transactions on Industrial Informatics, 2018.

[25] Z.C. Zacharia. Comparing and combining real and virtual experimentation: an effort to enhance students' conceptual understanding of electric circuits. Journal of Computer Assisted Learning, 23(2), 2007.

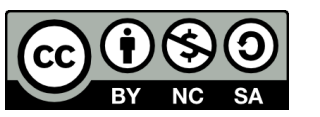
(c) 2019 by the authors. Submitted for possible open access publication under the terms and conditions of the Creative Commons Attribution CC BY-NC-SA 4.0 license (https://creativecommons.org/licenses/by-ncsa/4.0/deed.es). 